\title{
Treatment of Intraosseous Cystic Lesions of the Mandible by Conservative Enucleation and Cavity Filling with Allogenic Freeze-Dried Bone Mixed with Autologous Bone Marrow
}

\author{
Ye J, Liao J K and Chen WL* \\ Department of Oral and Maxillofacial Surgery, Sun Yat- \\ sen University, China \\ *Corresponding author: Wei-liang Chen, Department \\ of Oral and Maxillofacial Surgery, Sun Yat-sen Memorial \\ Hospital, Sun Yat-sen University, 107 Yan-jiang Road, \\ 510120 Guangzhou, China
}

Received: June 28, 2017; Accepted: August 01, 2017; Published: August 09, 2017

\begin{abstract}
Purpose: The aim of this study was to evaluate the outcome of defect filling with allogenic freeze-dried bone mixed with bone marrow following conservative enucleation of large intraosseous cystic lesions of the mandible.

Patients and Methods: Forty-two patients with large intraosseous cystic lesions of the mandible were treated by defect filling with allogenic freeze-dried bone combined with bone marrow following conservative cyst enucleation.

Results: All patients showed satisfactory healing. Dental rehabilitation including prosthesis and implant provision was successful in $10(23.8 \%)$ patients. The patients were followed for 13-48 (mean, 29.9) months using panoramic radiographic examination. Recurrence was seen in $2(11.2 \%)$ patients with KCOTs. Clinical and panoramic radiographic examination showed no residual or recurrent cyst in the remaining patients.
\end{abstract}

Conclusion: Allogenic freeze-dried bone mixed with autologous bone marrow may be as an alternative, viable filling material for intraosseous cystic lesions of the mandible removed by conservative enucleation.

Keywords: Intraosseous cystic lesion; Odontogenic cystic lesions; Enucleation; Allogenic freeze-dried bone; Autologous bone marrow

\section{Introduction}

Intraosseous cystic lesions, including dentigerous cysts, keratocystic odontogenic tumors (KCOTs), and aneurysmal bone cysts, are common lesions in the jaws. These lesions are found more frequently in the mandible [1]. Clinically, they may be asymptomatic or have acute or chronic findings, and swelling and pain may be the presenting symptoms. Intraosseous cystic lesions are evaluated by routine radiography, Computed Tomography (CT), and threedimensional CT. Biopsies may assist the diagnosis in selected cases. The use of a decalcified freeze-dried bone allograft is believed to enhance bone healing in large defects of the jaw occurring after cyst removal [2]. However, studies of defect filling using allogenic freezedried bone combined with bone marrow following enucleation of intraosseous cystic lesions have not yet been undertaken. Therefore, the aim of the present study was to evaluate the clinical outcomes of defect filling using allogenic freeze-dried bone combined with bone marrow following conservative enucleation of large intraosseous cystic lesions

\section{Patients and Methods}

Between January 2010 and May 2014, 42 patients with large intraosseous cystic lesions of the mandible were treated using a combination of allogenic freeze-dried bone and bone marrow to fill the defect following conservative cyst enucleation.
The study sample included 26 male and 16 female patients' aged 17-65 (mean, 40.5) years. Twenty-eight patients had dentigerous cysts, 11 had KCOTs and 3 had aneurysmal bone cysts; all diagnoses were confirmed by histological examination. Eighteen lesions were located in the right mandible, 16 were in the left mandible, and 8 encompassed both sides of the mandible. Radio graphically measured cystic lesion surface areas ranged from $4 \times 4 \mathrm{~cm}$ to $8 \times 4 \mathrm{~cm}$ (mean, $5.7 \times 4.4 \mathrm{~cm}$; Table 1). Teeth with cystic lesions were treated endodontically (Figure 1). All patients were evaluated postoperatively by repeated clinical examination by the operating surgeon. Radiographic examination was performed using panoramic radiographs taken preoperatively and immediately and 6 and 12 months postoperatively.

\section{Surgical procedure}

The patients were treated with conservative enucleation of large mandibular odontogenic cystic lesions using an intraoral approach under general anesthesia administered by nasotracheal intubation. First, a mucoperiostal flap was elevated to expose the outer surface of the cystic cavity. Prior to definitive surgery, biopsies of all cysts were taken to obtain histopathological diagnoses. The soft-tissue lining of the inner surface of the cystic cavity was carefully curettaged (Figure 2). The mandibular third molars or unerupted teeth with cystic lesions were removed; apicectomy was carried out and the inferior alveolar nerve was carefully protected during enucleation. Patients underwent bone marrow harvesting through the use of an 8-gauge
Austin J Surg - Volume 4 Issue 3 - 2017

ISSN : 2381-9030 | www.austinpublishinggroup.com

Chen et al. (C) All rights are reserved
Citation: Ye J, Liao JK and Chen WL. Treatment of Intraosseous Cystic Lesions of the Mandible by Conservative Enucleation and Cavity Filling with Allogenic Freeze-Dried Bone Mixed with Autologous Bone Marrow. Austin J Surg. 2017; 4(3): 1103. 


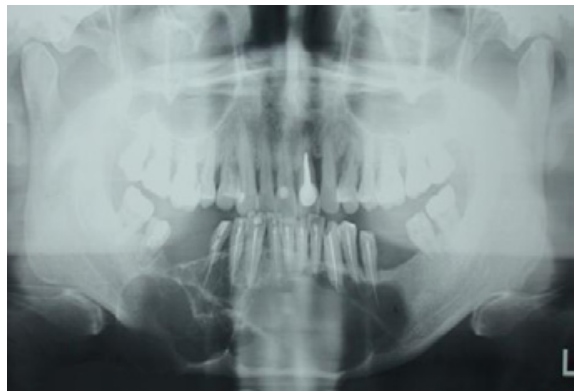

Figure 1: A 52-year-old man presented with a keratocystic odontogenic tumo of the mandible. preoperative panoramic radiograph showing an extensive radiolucent area from the right second premolar to the left first molar; teeth with cystic lesions were treated endodontically.

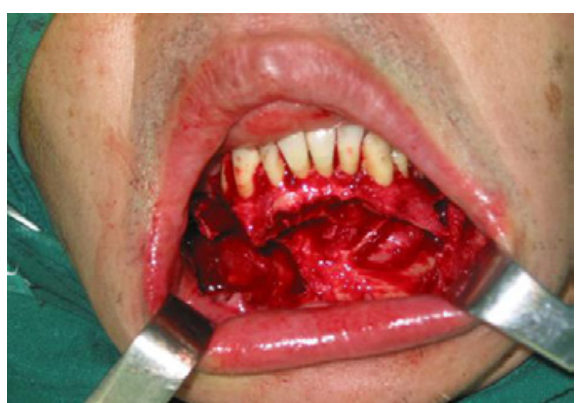

Figure 2: The cyst was enucleated and the body cavity was carefully curetted.

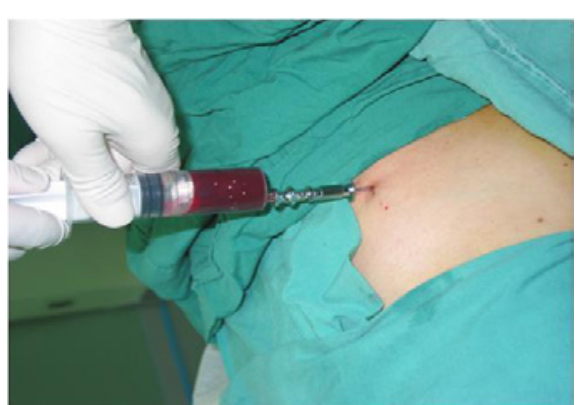

Figure 3: Patients underwent bone marrow harvesting through the use of an 8-gauge needle.

needle (Figure 3). Second, the cavity was filled completely by packing with Aorui (Shanxin Aorui Bio-material Co., Ltd., Taiyuan, China), a commercial product consisting of allogenic freeze-dried bone powder (particle diameters, $0.05-1 \mathrm{~mm}$ ), mixed with bone marrow taken from the iliac crest (Figure 3). Finally, the primary wound was closed; drainage was usually administered for 1 week postoperatively. Sutures were removed after 2 weeks. Dental prosthetic rehabilitation was undertaken 6 months after surgery when postoperative panoramic radiography showed complete absence of the lesion (Figures 4 \& 5). Straumann implants $(4.1 \times 10 \mathrm{~mm})$ were inserted into treated mandibular bone at 12 months after surgery.

\section{Results}

Teeth with cystic lesions were treated endodontically in 29 (69.0\%) patients and apicectomy was carried out in 19 (45.2\%) of these patients. The mandibular third molars, unerupted teeth, and teeth

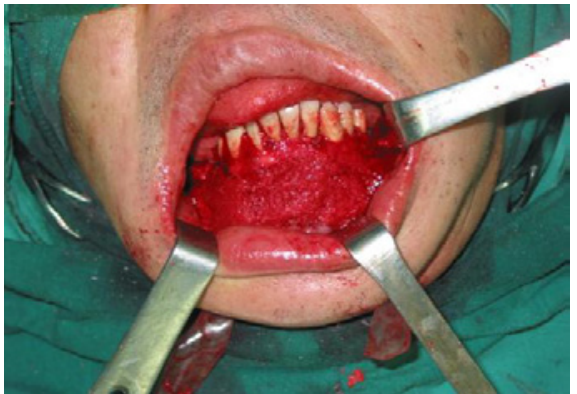

Figure 4: Filling materials for cysts. Allogenic freeze-dried bone powder and bone marrow were mixed and application of allogenic freeze-dried bone combined with bone marrow.
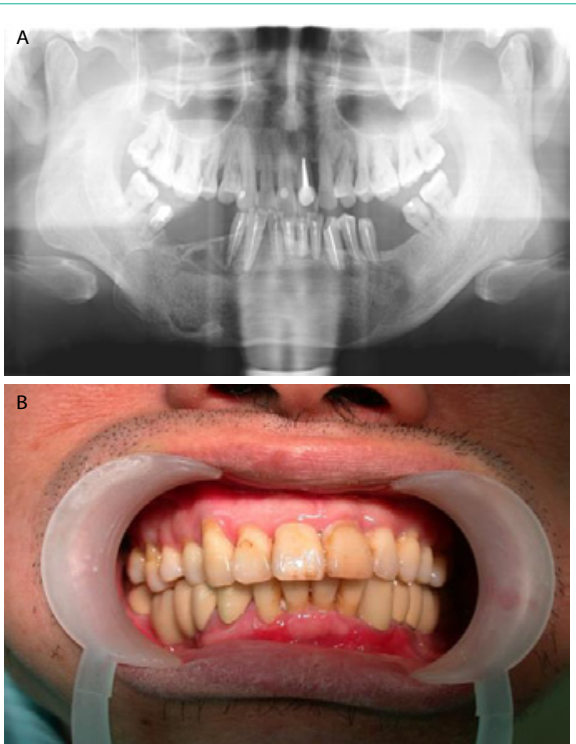

Figure 5: Six months after surgery. A) Panoramic radiograph showing complete absence of the lesion. B) Dental prosthetic rehabilitation was successful.

with cystic lesions were removed in 20 (47.6\%) cases. Postoperative hemorrhage occurred in one $(2.4 \%)$ patient. Wound dehiscence occurred in two (4.8\%) patients, and the wounds healed secondarily. All patients showed satisfactory healing. Dental rehabilitation including prosthesis and implant provision was successful in 10 (23.8\%) patients. Facial appearance and oral function were normal. The patients were followed for 13-48 (mean, 29.9) months using panoramic radiography. Recurrence was seen in $2(11.2 \%)$ patients with KCOTs (Table 1). Clinical and panoramic radiography showed no residual or recurrent cyst in the remaining patients.

\section{Discussion}

In the past few decades, numerous studies have investigated the treatment of jaw lesions using autogenous grafts, allogenous grafts, xenografts, or alloplastic and synthetic grafts as filling materials. In addition to reducing infection and healing disturbance, these materials have been used to accelerate bone regeneration, prevent soft-tissue collapse into the defect, and improve bone strength [3]. Autologous bone has repeatedly been reported to represent the gold standard of bone grafting, and the iliac crest is the most 
Table 1: Diagnosis, treatment, and clinical outcomes of 42 patients with intraosseous cystic lesions of the mandible.

\begin{tabular}{|c|c|c|c|c|c|c|c|}
\hline \multirow{2}{*}{ Case, age (years), sex } & \multirow{2}{*}{ Diagnosis } & \multirow{2}{*}{ Location } & \multirow{2}{*}{ Size $(\mathrm{cm})$} & \multirow{2}{*}{ Treatment } & \multirow{2}{*}{ Complications } & \multirow{2}{*}{$\begin{array}{l}\text { Follow-up } \\
\text { (months) }\end{array}$} & \multirow{2}{*}{ Outcome } \\
\hline & & & & & & & \\
\hline $1,52, \mathrm{M}$ & КСОТ & BM & $8 \times 4$ & $\mathrm{ET}+\mathrm{CEF}+\mathrm{TE}^{*}+\mathrm{AM}+\mathrm{DP}$ & None & 36 & NR \\
\hline $2,62, \mathrm{~F}$ & DC & RM & $5 \times 4$ & $\mathrm{ET}+\mathrm{CEF}$ & None & 13 & NR \\
\hline $3,18, M$ & $\mathrm{ABC}$ & RM & $5 \times 5$ & CEF & Hemorrhage & 24 & NR \\
\hline $4,24, M$ & DC & LM & $4 \times 4$ & $\mathrm{ET}+\mathrm{CEF}+\mathrm{TE} *$ & None & 19 & NR \\
\hline $5,40, \mathrm{M}$ & DC & LM & $6 \times 4$ & $\mathrm{ET}+\mathrm{CEF}+\mathrm{TE} \%$ & None & 38 & NR \\
\hline $6,36, M$ & DC & BM & $6 \times 4$ & $E T+C E F+T E *+A M$ & None & 48 & NR \\
\hline $7,22, \mathrm{~F}$ & DC & RM & $5 \times 4$ & $\mathrm{CEF}+\mathrm{TE} \mathrm{E}^{\star *}+\mathrm{DP}$ & None & 37 & NR \\
\hline $8,31, F$ & DC & RM & $4 \times 4$ & $\mathrm{ET}+\mathrm{CEF}$ & None & 20 & NR \\
\hline $9,20, F$ & KCOT & LM & $6 \times 4$ & $\mathrm{ET}+\mathrm{CEF}+\mathrm{TE}^{*}+\mathrm{AM}+\mathrm{DI}$ & None & 25 & NR \\
\hline $11,46, F$ & DC & LM & $5 \times 5$ & CEF & None & 33 & NR \\
\hline $12,49, \mathrm{~F}$ & DC & LM & $5 \times 4$ & CEF & None & 17 & $N R$ \\
\hline $13,38, M$ & DC & RM & $6 \times 5$ & $\mathrm{ET}+\mathrm{CEF}+\mathrm{TE}^{\#}$ & None & 42 & NR \\
\hline $14,56, \mathrm{M}$ & DC & $\mathrm{BM}$ & $8 \times 5$ & $\mathrm{ET}+\mathrm{CEF}+\mathrm{TE}^{*}+\mathrm{AM}+\mathrm{DP}$ & None & 26 & NR \\
\hline $15,49, \mathrm{~F}$ & KCOT & LM & $4 \times 4$ & CEF & None & 33 & NR \\
\hline $16,19, \mathrm{M}$ & DC & RM & $5 \times 5$ & $\mathrm{ET}+\mathrm{CEF}+\mathrm{AM}$ & None & 27 & NR \\
\hline $17,51, \mathrm{M}$ & КСOT & LM & $6 \times 4$ & $\mathrm{ET}+\mathrm{CEF}+\mathrm{TE}{ }^{\star} * \mathrm{AM}+\mathrm{DP}$ & None & 40 & NR \\
\hline $18,44, \mathrm{M}$ & КСOT & BM & $7 \times 4$ & $E T+C E F+A M$ & None & 33 & Recurrent ${ }^{\boldsymbol{\Delta}}$ \\
\hline $19,56, F$ & KCOT & RM & $6 \times 4$ & $\mathrm{CEF}+\mathrm{TE}^{\star *}+\mathrm{DP}$ & None & 19 & NR \\
\hline $20,53, M$ & KCOT & LM & $6 \times 4$ & $\mathrm{ET}+\mathrm{CEF}+\mathrm{TE}^{*}+\mathrm{AM}+\mathrm{DI}$ & None & 26 & NR \\
\hline $21,49, \mathrm{M}$ & DC & RM & $5 \times 4$ & $\mathrm{CEF}+\mathrm{TE} \%$ & None & 32 & NR \\
\hline $24,53, \mathrm{M}$ & KCOT & RM & $6 \times 6$ & $\mathrm{ET}+\mathrm{CEF}+\mathrm{AM}$ & None & 38 & NR \\
\hline $25,36, F$ & DC & RM & $4 \times 4$ & CEF & None & 24 & NR \\
\hline $26,51, M$ & DC & LM & $7 \times 4$ & $\mathrm{ET}+\mathrm{CEF}+\mathrm{TE} *+\mathrm{AM}$ & None & 28 & NR \\
\hline $27,29, \mathrm{M}$ & KCOT & $\mathrm{BM}$ & $8 \times 4$ & $E T+C E F+T E^{\star *}+A M+D P$ & None & 36 & NR \\
\hline $28,47, F$ & DC & RM & $6 \times 4$ & $\mathrm{ET}+\mathrm{CEF}+\mathrm{TE}^{\#}$ & Wound dehiscence & 18 & NR \\
\hline $29,56, M$ & KCOT & LM & $6 \times 5$ & $\mathrm{ET}+\mathrm{CEF}+\mathrm{AM}$ & None & 26 & NR \\
\hline $30,38, F$ & DC & RM & $5 \times 4$ & CEF & None & 42 & $N R$ \\
\hline $31,43, \mathrm{M}$ & KCOT & RM & $5 \times 5$ & $\mathrm{ET}+\mathrm{CEF}$ & None & 32 & NR \\
\hline $32,27, M$ & $\mathrm{DC}$ & LM & $5 \times 4$ & $\mathrm{ET}+\mathrm{CEF}$ & None & 28 & NR \\
\hline $33,17, F$ & $\mathrm{ABC}$ & RM & $6 \times 4$ & CEF & None & 16 & NR \\
\hline $34,47, \mathrm{M}$ & DC & BM & $5 \times 4$ & $\mathrm{ET}+\mathrm{CEF}+\mathrm{AM}$ & None & 35 & NR \\
\hline $35,26, M$ & DC & RM & $6 \times 4$ & $\mathrm{ET}+\mathrm{CEF}+\mathrm{TE} *+\mathrm{AM}$ & None & 14 & NR \\
\hline $36,37, F$ & KCOT & LM & $7 \times 5$ & $\mathrm{ET}+\mathrm{CEF}+\mathrm{TE}^{*}+\mathrm{AM}+\mathrm{DP}$ & None & 25 & NR \\
\hline $37,37, \mathrm{M}$ & KCOT & RM & $6 \times 4$ & $\mathrm{ET}+\mathrm{CEF}+\mathrm{AM}$ & None & 36 & NR \\
\hline $38,42, \mathrm{M}$ & KCOT & LM & $7 \times 4$ & $\mathrm{ET}+\mathrm{CEF}+\mathrm{TB}+\mathrm{DP}$ & None & 43 & NR \\
\hline $39,47, F$ & DC & $\mathrm{BM}$ & $6 \times 4$ & $\mathrm{ET}+\mathrm{CEF}+\mathrm{TE} \mathrm{E}^{\#}$ & None & 33 & NR \\
\hline $40,45, M$ & DC & LM & $5 \times 4$ & CEF & None & 25 & NR \\
\hline $41,65, M$ & DC & $\mathrm{BM}$ & $8 \times 4$ & $\mathrm{ET}+\mathrm{CEF}+\mathrm{TE}^{*}+\mathrm{AM}+\mathrm{DI}$ & None & 21 & NR \\
\hline $42,36, \mathrm{M}$ & DC & LM & $4 \times 4$ & CEF & None & 32 & $N R$ \\
\hline
\end{tabular}

M: Male; F: Female; KCOT: Keratocystic Odontogenic Tumor; DC: Dentigerous Cysts; ABC: Aneurysmal Bone Cysts; BM: Bilateral Mandible; LM: Left Mandible; RM: Right Mandible; ET: Endodontic Treatment; CEF: Conservative Enucleation and Filling with allogenic freeze-dried bone mixed with autologous bone marrow; TE: Tooth Extraction; AM: Apicectomy; DP: Dental Prosthesis; DI: Dental Implant; NR: No Recurrence.

*teeth with cystic lesions; *unerupted teeth; " ${ }^{*}$ mandibular third molars. ${ }^{\wedge}$ Recurrent KCOTs were small lesions and could be treated by simple excision. 
frequently chosen donor site because the iliac spongiosa provides osteoconductive, osteoinductive, and osteogenic properties [4]. However, clinical reports of jaw defect filling with autogenous iliac bone after cyst enucleation are rare.

One study showed that bone density was significantly greater in patients with cystic lesions of the jaws who underwent enucleation and bone grafting with demineralized freeze-dried bone allografts than in those whose defects were packed with an absorbable gelatin sponge. This finding demonstrated that decalcified freeze-dried bone allografts enhanced bone healing in jaw defects after cyst removal [2]. Horowitz et al. [5] reported the use of Kiel bone xenografts in combination with autologous aspirated bone marrow to graft 20 cystic jaw defects. Sixteen (80\%) cases were successful. Results were evaluated by clinical and radiographic follow-up of the patients for up to 4 (average, 2.3) years after surgery. Causes of failure were reported to be the inability to create a water-tight closure, followed by immediate secondary infection, rather than host rejection [5]. The use of mineralized bone substitutes, such as $\beta$-tricalcium phosphate or hydroxyapatite, leads to complete ossification of large defects after 12 months [6]. In the present study, facial appearance and oral function were normal. Two cases of KCOT cyst recurrence were observed at 33 and 42 months postoperatively. Clinical and panoramic radiography showed no residual or recurrent cyst in the remaining patients. We believe that defect filling using allogenic freeze-dried bone combined with bone marrow following conservative enucleation is a simple, safe, and reliable alternative treatment for large intraosseous cystic lesions, including dentigerous cysts, KCOTs, and aneurysmal bone cysts.

The treatment of cystic jaw lesions using a combination of allogenic freeze-dried bone and bone marrow reduces infection and healing disturbance, accelerates bone regeneration, prevents soft-tissue collapse, and improves bone strength. The preferred treatment of these lesions is cyst enucleation, and the resultant cavity can be filled with different materials to accelerate bone healing and provide an adequate quantity and quality of regenerated bone for the subsequent placement of prostheses or implants. Our results showed that facial and oral function were normal in all patients and that dental rehabilitation, including prosthesis and implant provision, was successful in $10(23.8 \%)$ patients. Accelerated bone healing is due to the osteoinductive and osteoconductive effects of allogenic freezedried bone, which contains osteoinductive proteins $[7,8]$. Ondrus et al. [9] reported that the application of tricalcium phosphate mixed with autologous bone marrow can achieve better and faster healing of benign bone lesions than the application of tricalcium phosphate granules alone.

The World Health Organization has described the tendency of an odontogenic keratocyst to progress to a KCOT based on the wellknown aggressive behavior of this lesion, its histology, and new genetic information [10]. KCOTs comprise approximately $11 \%$ of all cysts of the jaws [11]. Madras et al. [10] reported that the $29 \%$ recurrence rate in their case series was consistent with previously established data, and all recurrence occurred within 2 years post-intervention. In our case series, recurrence was observed at 33 and 43 months in 2 (11.2\%) patients with KCOTs treated with enucleation. The recurrent KCOTs were small lesions and could be treated by simple excision. In our study, intraosseous cystic lesion of developmental origin presented with malignant degeneration.

The wounds were monitored daily for primary wound healing. Two (4.8\%) patients developed wound dehiscence, which may have been due to excessive tension and premature suture removal, and the wounds healed secondarily. Wound closure with less tension and delayed suture removal may prevent wound dehiscence.

The results of this study support the clinical application of allogenic freeze-dried bone mixed with autologous bone marrow as an alternative, viable filling material for intraosseous cystic lesions of the mandible removed by conservative enucleation.

\section{References}

1. Iatrou I, Theologie-Lygidakis N, Leventis M. Intraosseous cystic lesions of the jaws in children: a retrospective analysis of 47 consecutive cases. Oral Surg Oral Med Oral Pathol Oral Radiol Endod. 2009; 107: 485-492.

2. Bodner L. Effect of decalcified freeze-dried bone allograft on the healing of jaw defects after cyst enucleation. J Oral Maxillofac Surg. 1996; 54: 12821286.

3. Ettl T, Gosau M, Sader R, Reichert TE. Jaw cysts - Filling or no filling after enucleation? A review. J Craniomaxillofac Surg. 2012; 40: 485-493.

4. Giannoudis PV, Dinopoulos H, Tsiridis E. Bone substitutes: an update. Injury. 2005; 36: S20-27.

5. Horowitz I, Bodner L. Use of xenograft bone with aspirated bone marrow for treatment of cystic defect of the jaws. Head Neck. 1989; 11: 516-523.

6. Velich N, Németh Z, Hrabák K, Suba Z, Szabó G. Repair of bony defect with combination biomaterials. J Craniofac Surg. 2004; 15: 11-15.

7. Traianedes K, Russell JL, Edwards JT, Stubbs HA, Shanahan IR, Knaack D. Donor age and gender effects on osteoinductivity of demineralized bone matrix. J Biomed Mater Res B Appl Biomater. 2004; 70: 21-29.

8. Wang JC, Alanay A, Mark D, Kanim LE, Campbell PA, Dawson EG, et al. A comparison of commercially available demineralized bone matrix for spinal fusion. Eur Spine J. 2007; 16: 1233-1240.

9. Ondruš S, Straka M, Bajerová J. Tricalcium phosphate mixed with autologous bone marrow in the treatment of benign cystic bone lesions in children. Acta Chir Orthop Traumatol Cech. 2011; 78: 544-550.

10. Madras J, Lapointe $\mathrm{H}$. Keratocystic odontogenic tumour: reclassification of the odontogenic keratocyst from cyst to tumour. J Can Dent Assoc. 2008; 74: 165-165h.

11. Maurette PE, Jorge J, de Moraes M. Conservative treatment protocol of odontogenic keratocyst: a preliminary study. J Oral Maxillofac Surg. 2006; 64: 379-383.
Austin J Surg - Volume 4 Issue $3-2017$
ISSN : 2381-9030 | www.austinpublishing group.com
Chen et al. @ All rights are reserved
Citation: Ye J, Liao JK and Chen WL. Treatment of Intraosseous Cystic Lesions of the Mandible by Conservative Enucleation and Cavity Filling with Allogenic Freeze-Dried Bone Mixed with Autologous Bone Marrow. Austin J Surg. 2017; 4(3): 1103 . 